\title{
Method to Convert an 8-item PCL-5 Score to a Total PCL-5 Score: A Scaled Equation Analysis
}

*For correspondence:

Matthew.Price@uvm.edu (MP)

Present address: 2 Colchester Ave, Burlington VT 05405

\section{Zoe Brier ${ }^{1}$ and Matthew Price ${ }^{1 *}$}

${ }^{1}$ Center for Research on Emotion, Stress, and Technology, Department of Psychological Science, University of Vermont

Abstract The repeated assessment of PTSD symptom severity is a critical component of research and evidence-based interventions. PTSD is comprised of 20 symptoms across four clusters. However, 20-item measures can be burdensome to administer frequently, especially in ecological momentary assessment (EMA) where multiple assessments are conducted per day. An abbreviated 8-item version of the PCL-5 was developed to obtain information about PTSD symptoms at reduced effort to the participant. However, the symptom severity scale by which symptoms can range between the abbreviated (0-32) and full (0-80) scales differ, which limits the interpretability of the abbreviated scale. The current study evaluated three different methods (a proportional, linear regression, and genetic programming model) to convert scores from the abbreviated measure to that of the full PCL. These equations were then compared across two testing samples to assess their accuracy and bias. The genetic programming and linear regression equation were superior to a proportional equation in both measures of accuracy and bias, with the genetic programming equation demonstrating less bias overall than the linear regression. Further, the diagnostic utility of each method was found to be similar. These results provide a method to convert abbreviated PCL scores to that of the full scale, which increases the utility of this abbreviated scale.

\section{Measure Availability}

Copies of the Abbreviated PCL-5 (4 and 8 item version) are found here:

https://www.crestresearch.org/research/abbreviated-pcl

\section{Introduction}

Posttraumatic Stress Disorder (PTSD) is a major public health concern, with approximately 8.3\% of adults in the United States having met criteria for the disorder in their lifetime and lifetime exposure to potentially traumatic events (PTEs) for adults estimated at 89.7\% (Kilpatrick et al., 2013). Recent investigations of PTSD have suggested that symptom severity fluctuates considerably within a day, possibly due to responses to environmental cues (Gelkopf, Pickman, Carlson, Greene, 2019). Understanding these symptom dynamics is necessary to improve our diagnosis and treatment of 
PTSD. In order to examine these changes in PTSD symptom severity, it is necessary to conduct multiple assessments within a brief period as in an ecological momentary assessment design (EMA; Shiffman Stone, 2008). Recent research examined the in vivo assessment of PTSD symptoms post-trauma and found EMA to be a feasible such method (Price, van Stolk-Cooke, Legrand, et al., 2018) and, in fact, may even be therapeutic (Dewey et al., 2015). The widespread availability of mobile devices capable of administering PTSD assessments has made such research more feasible in recent years. However, a key challenge to conducting such work are assessments suitable for such frequent assessments.

PTSD is comprised of 20 symptoms across four clusters [intrusion symptoms, avoidance, negative alterations in cognition and mood (NACM), and alterations in arousal and reactivity (AAR)] (APA, 2013). Validated PTSD measures, such as the PTSD Symptom Checklist for the DSM-5 (PCL-5; Weathers, Litz, et al., 2013), assess all 20 symptoms. The PCL-5 assesses both the type and severity of PTSD symptoms on a Likert Scale ( 0 = not at all, $4=$ extremely). The PCL-5 has demonstrated high reliability and validity in several populations including trauma-exposed college students (Blevins, Weathers, Davis, Witte, Domino, 2015) and militarypopulations (Bovin et al., 2016; Wortmann et al., 2016).

The length of the PCL-5 is a limitation when used as a screening instrument or when it is administered frequently (Lang et al., 2005; Price et al., 2016). In primary care and other outpatient settings, providers often have limited time to assess individuals (Duhoux et al., 2013). Tools have been developed to assess mental health conditions efficiently (see Arroll et al., 2009; Löwe, Kroenke, Gräfe, 2005; Prins et al., 2016). These measures range from 2 to 6 items, which highlights how there is a preference for abbreviated measures in these settings. EMA designs recommend that assessments be brief to increase compliance and the validity of the data collected (Shiffman, Stone, Hufford, 2008). EMA is an in vivo assessment method in which participants complete assessments in real time at a high frequency. EMA studies with PTSD have administered assessments as frequently as every 45 minutes over a period of days (Shapiro, Jamner, Davydov, James, 2002). A recent study conducted by Schuler and colleagues (2019) examined PTSD symptoms assessed retrospectively over a week compared with EMA assessment. Findings indicated retrospective assessment to be closer to the worst day of assessment, rather than the average score over the week. This implies that EMA may be an effective method of assessment of PTSD symptoms. Completing a 20-item measure frequently may prove overly burdensome resulting in lower compliance with data collection.

EMA guidelines recommend allowing less than five minutes per assessment (Price, van Stolk- Cooke, Brier, Legrand, 2018). Abbreviated measures for other diagnoses have been developed to address this problem. For example, the Patient Health Questionnaire has a 2-item version (PHQ-2; Löwe, Kroenke, Gräfe, 2005) that can accurately assess and screen for depression symptoms. This measure also suggests that it is possible to obtain an accurate measure of symptoms without having to assess all possible symptoms of a diagnosis.

A short-form 8-item version of the PCL-5 was developed for administering EMA studies on PTSD (Price, Szafranski, van Stolk-Cooke, Gros, 2016). Two short-forms were developed, an 8 -item version to assess symptom severity and a 4-item version used as a screening instrument. The benefit of these assessments is the reduced length of administration. The measure demonstrated comparable psychometric properties to the PCL-5 and scores on the abbreviated version were highly correlated with that of the total scores (Pereira-Lima et al., 2019). However, the 8-item abbreviated scale had scores that ranged from 0-32 whereas the total PCL-5 scale had scores that ranged from 0-80. The difference in scales make it difficult to interpret scores on the abbreviated scale, and thus limits the utility of this measure. A method to convert the scores of the abbreviated measure to the full scale would improve the utility of the former. 
There are several possible methods to scale the abbreviated PCL-5 score including a proportion scale of item-level weighting. It is unclear, however, which will best approximate the full score across individuals. The two measures are highly correlated, which suggests that a proportional relationship may exist. A proportional relationship, however, implies that all symptoms of PTSD contribute to the total score equally. Alternatively, the contribution of certain symptoms to overall severity may vary such that weighting specific symptoms would result in a more accurate score. Recent work examining the symptom network of PTSD has demonstrated that certain symptoms are more associated with overall symptom severity and presentation (Bryant et al., 2017; Price, Legrand, Brier, Hebert-Dufresne, 2019). There are several methods by which to obtain weights including OLS (Ordinary Least Squares) regression or more data driven approaches such as machine learning. These different approaches should be compared to determine how best to scale the abbreviated PCL-5 scores.

The present study aimed to determine the optimal method to convert scores on the abbreviated PCL 8-item version to the full PCL-5. Three methods were compared: 1) a proportional scaling that used the same weights for all items, 2) a linear regression based scaling using weights from an OLS regression and 3) a machine learning approach in which weights were evolved using genetic programming. The PCL 8-item was of interest as this was intended to batter gauge symptom severity. It was hypothesized that a linear regression and genetic programming method would be superior to proportional scaling. The performances of the scaled models were also evaluated by comparing the scaled total scores to the full PCL- 5 total scores and evaluating the diagnostic utility. The present study consisted of three experiments. The first used data from a community-exposed sample to derive and evaluate the weights. The second cross-validated the performance of the weights in a new set of data obtained from a community sample. The third also cross-validated the performance of the weights in a clinical sample as well as the diagnostic utility of the scaled score.

\section{Experiment 1}

\section{Methods}

\section{Participants}

Participants were 1151 trauma-exposed adults recruited through Amazon's Mechanical Turk (MTurk). MTurk is an online crowdsourcing platform from which valid and reliable clinical data can be collected (Shapiro, Chandler, Mueller, 2013; van Stolk-Cooke et al., 2018). For this study, 1600 Human Intelligence Tasks (HITs) were made available to participants with keywords survey, stress, gender, women/men, and health, in which half of the HITs were designated for each gender. Participants in this study were able respond to the HIT only if they had a previous HIT success rate of $70 \%$ and an IP address in the United States. Participants were included if they experienced a Criterion A traumatic event (APA, 2013). Participants received five validity checks within the online survey (e.g., "For this question, please select choice 3"). Participants were included if they answered at least three validity questions correctly and completed the survey in greater than five minutes (Figure 1).

Participants ages ranged from $18-74(M=31.74, S D=11.12)$, and $56.7 \%$ of the sample was female. The majority of the sample identified as White (83.2\%), 7.07.2\% as Asian American, $0.3 \%$ as Pacific Islander/Native Alaskan, $1.1 \%$ as American Indian, $0.9 \%$ as Bi-racial, and $0.4 \%$ as another race. A fifth of the sample (20.9\%) identified as Hispanic. The majority of the sample (76.2\%) reported an annual income of $\$ 30,000$ or less, $16.2 \%$ completed high school as their highest education level, and $45.3 \%$ completed college or had more education (Table 1). 
Figure 1. Histogram matrix of bias and accuracy for each model in Experiment 1.

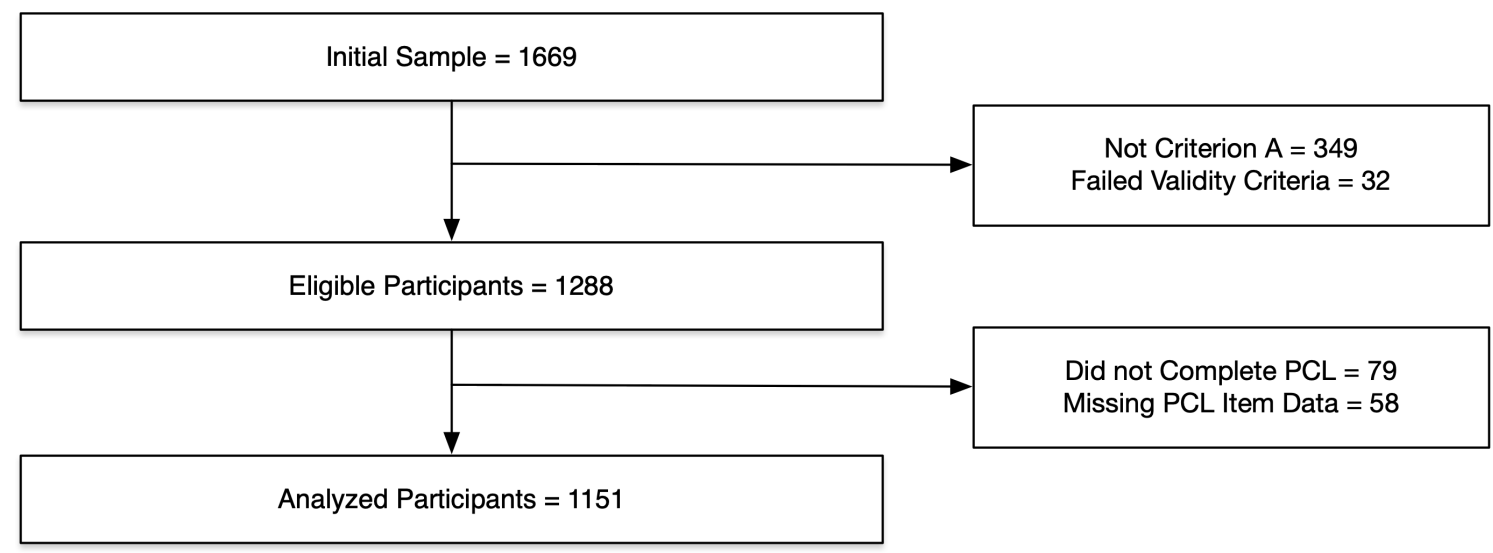

Measures

Life Events Checklist extended version (LEC; Weathers, Blake, et al., 2013). The LEC extended version is a 25 -item self-report measure used to assess potential Criterion A traumatic events. The LEC assesses trauma based on the type of event (e.g. natural disaster) and its nature by inquiring if the event happened to the individual, they witnessed it, they learned about it, or it doesn't apply to the individual. The LEC also asks individuals to identify the worst event that they experienced, and further assesses the nature of this event to determine if it meets Criterion A.

PTSD Checklist for DSM-5 (PCL-5; Weathers, Litz, et al., 2013). The PCL-5 is a 20- item self-report measure which assesses type and severity of PTSD related symptoms. The PCL-5 is measured on a 0-4 Likert scale ( $0=$ Not at all, $4=$ Extremely). The PCL-5 was anchored to the most severe trauma identified in the LEC. The PCL-5 had high internal consistency $(=0.95)$. The abbreviated 8-item version of the PCL-5 that was used in this study (Price et al., 2016) includes the items from the original measure listed in Table 2. The administration time of the full PCL-5 is approximately 5-10 minutes (Weathers, Litz, et al., 2013). Therefore, the administration time of the abbreviated version is approximately 2-4 minutes.

\section{Data Analytic Plan}

Analyses were conducted using R version 3.5.1. Participants $(N=58)$ were excluded if they did not have complete data on the items in the PCL-8. Three models were examined in order to assess the best fit to the original PCL-5. For each model, the mean absolute value and actual value of the difference between the PCL-8 scaled score and the PCL-5 score were calculated to assess the accuracy and bias of the model, respectively. Values closer to 0 indicate better approximation of the total score. A proportional model in which the abbreviated total score was scaled by a factor of 2.5 was evaluated.

$$
2.5\left(P C L 8_{\text {total }}\right)=P C L_{\text {proportional }}
$$

A linear regression model was evaluated next (Table 3). This equation was created using multiple linear regression in which the PCL- 5 total score was regressed on all PCL-8 items. These regression coefficients were used as item weights. 
Table 1. Demographics of each experiment and full sample

\begin{tabular}{|c|c|c|c|c|}
\hline & Experiment 1 & Experiment 2 & Experiment 3 & Total \\
\hline N & 1151 & 181 & 89 & 1421 \\
\hline Mean Age (SD) & $31.74(11.12)$ & $31.62(11.69)$ & 36.9 (10.19) & $32.05(11.20)$ \\
\hline \multicolumn{5}{|l|}{ Gender } \\
\hline Female (\%) & 56.7 & 61.7 & 43.8 & 43.4 \\
\hline Male (\%) & 43.3 & 38.3 & 55.1 & 56.5 \\
\hline Other (\%) & - & - & 1.1 & 0.1 \\
\hline \multicolumn{5}{|l|}{ Race } \\
\hline African-American (\%) & 7.0 & 5.0 & 2.3 & 6.4 \\
\hline American Indian (\%) & 1.1 & 0.6 & 4.5 & 1.2 \\
\hline Asian-American (\%) & 7.2 & 3.9 & 2.3 & 6.5 \\
\hline Bi-racial (\%) & 0.9 & 4.4 & 3.4 & 1.5 \\
\hline Pacific Islander (\%) & 0.3 & 0.0 & 1.1 & 0.3 \\
\hline Other race (\%) & 0.4 & 1.6 & 0.0 & 0.5 \\
\hline White (\%) & 83.2 & 84.4 & 86.4 & 83.6 \\
\hline \multicolumn{5}{|l|}{ Ethnicity } \\
\hline Hispanic / Latino (\%) & 20.9 & 6.8 & 3.6 & 82.0 \\
\hline \multicolumn{5}{|l|}{ Income } \\
\hline$\leq \$ 30,000$ annually $(\%)$ & 76.2 & 27.4 & 88.8 & 70.8 \\
\hline$\geq \$ 30,000$ annually (\%) & 23.8 & 72.6 & 11.2 & 29.1 \\
\hline \multicolumn{5}{|l|}{ Education } \\
\hline Completed High School (\%) & 16.2 & 12.8 & 29.2 & 16.6 \\
\hline Completed College (\%) & 45.3 & 45.3 & 8.9 & 43.0 \\
\hline
\end{tabular}

$$
\begin{gathered}
2.11\left(P C L_{1}\right)+1.82\left(P C L_{4}\right)+1.54\left(P C L_{6}\right)+1.69\left(P C L_{7}\right)+2.92\left(P C L_{9}\right)+ \\
2.96\left(P C L_{1} 2\right)+2.59\left(P C L_{1} 8\right)+2.49\left(P C L_{1} 9\right)=P C L_{\text {Regression }}
\end{gathered}
$$

Symbolic regression via genetic programming (GP) was used to develop the third equation. GP is an evolutionary algorithm in which a set of candidate solutions to a problem are evolved over a series of generations (Schmidt Lipson, 2009). Applied to the current study, GP was used to minimize the difference between the obtained PCL-5 total score and the estimated PCL-5 total score from the PCL-8. The analyses were conducted in Eureqa (Boston, MA). Eureqa is a GP that seeks to minimize prediction error (i.e., the absolute difference between the estimated and actual score) and the complexity of the solution (i.e., the number of terms in the model). For the current study, the GP was allowed to select from basic arithmetic operations (addition, subtraction, multiplication, exponents), co-evolved numerical constants to serve as coefficients, and variables corresponding to the items from the PCL-8. Ten de novo runs of Eureqa were used, each starting from a random initial population of equations to evaluate consistency across the non-dominated solutions. Each run was terminated when Eureqa's internal heuristic of coverage reached $100 \%$, suggesting it was unlikely that a superior solution would be found with continued run time. Within each evaluation, a train/test split of 70\%/30\% of the data was used. The optimal equation from each run was recorded and the mean coefficient for each variable in the solution was used to create the final equation. 
Table 2. Item comparison of the full PTSD Checklist for DSM-5 (PCL-5) and the abbreviated PCL-5 8-item version

\begin{tabular}{|c|c|c|}
\hline Item in PCL-8 & Item in PCL-5 & Question Text \\
\hline 1 & 1 & $\begin{array}{l}\text { Repeated, disturbing, and unwanted memories } \\
\text { of the stressful experience? }\end{array}$ \\
\hline 2 & 4 & $\begin{array}{l}\text { Feeling very upset when something reminded } \\
\text { you of the stressful experience? }\end{array}$ \\
\hline 3 & 6 & $\begin{array}{l}\text { Avoiding memories, thoughts, or feelings related } \\
\text { to the stressful experience? }\end{array}$ \\
\hline 4 & 7 & $\begin{array}{l}\text { Avoiding external reminders of the stressful experience } \\
\text { (for example, people, places, conversations, } \\
\text { activities, objects, or situations)? }\end{array}$ \\
\hline 5 & 9 & $\begin{array}{l}\text { Having strong negative beliefs about yourself, other people, } \\
\text { or the world (for example, having thoughts such as: I am } \\
\text { bad, there is something seriously wrong with me, no one } \\
\text { can be trusted, the world is completely } \\
\text { dangerous)? }\end{array}$ \\
\hline 6 & 12 & Loss of interest in activities that you used to enjoy? \\
\hline 7 & 18 & Feeling jumpy or easily startled? \\
\hline 8 & 18 & Having difficulty concentrating? \\
\hline
\end{tabular}

$$
\begin{gathered}
2.15\left(P C L_{1}\right)+1.78\left(P C L_{4}\right)+1.79\left(P C L_{6}\right)+1.35\left(P C L_{7}\right)+3.03\left(P C L_{9}\right)+ \\
2.96\left(P C L_{1} 2\right)+2.88\left(P C L_{1} 8\right)+2.53\left(P C L_{1} 9\right)=P C L_{G P}
\end{gathered}
$$

\section{Results}

\section{Proportional Model}

The accuracy of the proportional model was $\mathrm{M}=3.88$ points $(95 \% \mathrm{Cl}[3.67,4.09])$. The bias was $\mathrm{M}=-2.66$ points, $95 \% \mathrm{Cl}[-2.93,-2.40]$. This difference suggests this model was likely to underestimate the actual score (Figure 2).

\section{Linear Regression Model}

The accuracy of the regression model yielded a mean of 2.68 points $95 \% \mathrm{Cl}[2.53,2.83]$ and a bias of $\mathrm{M}=0.41$ points $95 \% \mathrm{Cl}[0.20,0.63]$.

\section{Genetic Programming Model}

The accuracy of the GP model was $\mathrm{M}=2.66$ points, 95 was $\mathrm{M}=0.09$ points, $95 \% \mathrm{Cl}[-.13$, .31]. This difference suggests the scaled score does not consistently over or under estimate the actual score.

These three models suggested that the linear regression and GP scaling equations were superior to a proportional equation in this initial dataset. The GP model performed better than the linear regression equation in bias of the models. Therefore, these three models were assessed in 
Figure 2. Histogram matrix of bias and accuracy for each model in Experiment 1.
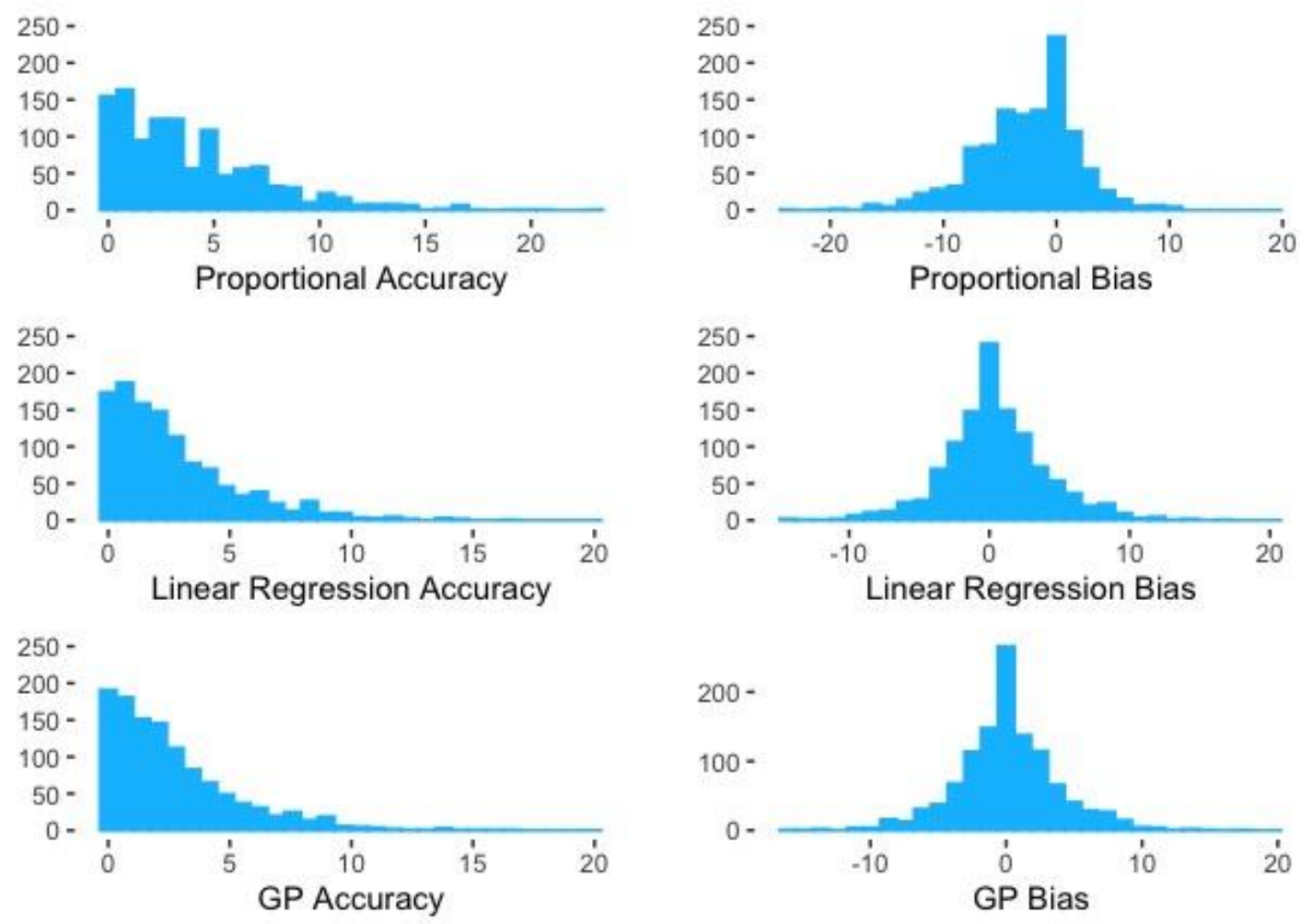

two unseen datasets to guard against overfitting (Whelan Garavan, 2014).

\section{Experiment 2}

\section{Methods}

Participants

Participants in this sample were 181 trauma exposed individuals recruited via Amazon's Mechanical Turk using the same procedure as described previously. These participants were held out from the initial dataset for cross-validation. Participants' ages in this sample ranged from 18 to $72(\mathrm{M}=31.62, \mathrm{SD}=11.69)$ and $61.7 \%$ identified as female. The majority of participants identified as White (84.4\%), 5.0\% identified as African American, 3.9\% identified as Asian American, 0.6\% as American Indian, $4.4 \%$ as Bi-Racial, and $1.6 \%$ as Other. The majority of participants (93.2\%) identified as Not Hispanic. In this sample, $12.8 \%$ completed high school as their highest education level and $45.3 \%$ completed college or had more education. About a quarter of participants (27.4\%) reported that their annual income was $\$ 30,000$ per year or less. Inclusion criteria for this study was identical to the previous sample (Table 1). Participants in this study completed self-report measures, including the LEC and PCL-5 described previously in the original sample. Three participants were excluded from these analyses as they did not have complete data on the PCL-8 (Figure 3).

\section{Results}

The performance of each model described previously was examined in this dataset for both difference between the scaled scores and full PCL-5 scores (absolute value), as well as bias 
Figure 3. Consort diagram of the Experiment 2.

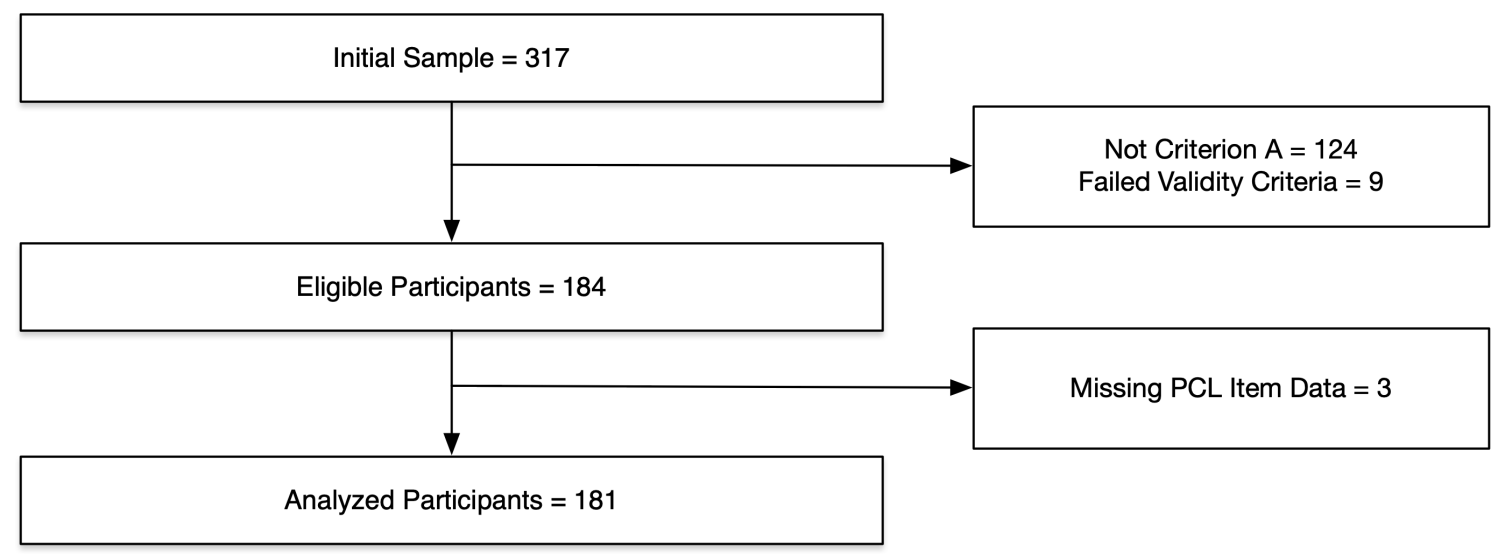

(actual differences) (Table 4). The linear regression $\left(M_{\text {accuracy }}=3.92,95 \% \mathrm{Cl}[3.40,4.45] ; M_{\text {bias }}=-.32\right.$, $95 \% \mathrm{Cl}[-1.10, .45])$ and GP models $\left(M_{\text {accuracy }}=4.02,95 \% \mathrm{Cl}[3.47,4.56] ; M_{\text {bias }}=-.82,95 \% \mathrm{Cl}[-1.61\right.$, .03]) both performed more accurately and with less bias than the proportional model ( $M_{\text {accuracy }}=$ $\left.5.15,95 \% \mathrm{Cl}[4.41,5.90] ; M_{\text {bias }}=-3.24,95 \% \mathrm{Cl}[-4.19,-2.29]\right)$. Both the linear regression and GP model had comparable accuracy, and the linear regression model had less bias than the GP model (Table 4).

Table 3. Accuracy and bias of equations in each experiment

\begin{tabular}{lcc}
\hline & Proportional Model \\
& Accuracy - M $[95 \% \mathrm{Cl}]$ & Bias - M [95\% Cl] \\
Experiment 1 & $3.88[3.67,4.09]$ & $-2.66[-2.93,-2.40]$ \\
Experiment 2 & $5.15[4.41,5.90]$ & $-3.24[-4.19,-2.29]$ \\
Experiment 3 & $4.49[3.75,5.23]$ & $-3.22[-4.22,-2.23]$ \\
\hline & Linear Regression Model \\
& Accuracy - M [95\% Cl] & Bias - M [95\% Cl] \\
Experiment 1 & $2.68[2.53,2.83]$ & $0.41[0.20,0.63]$ \\
Experiment 2 & $3.92[3.40,4.45]$ & $-0.32[-1.10,0.45]$ \\
Experiment 3 & $3.42[2.82,4.02]$ & $0.57[-0.37,1.50]$ \\
\hline & GP Model & \\
& Accuracy - M $[95 \% \mathrm{Cl}]$ & Bias - M $[95 \% \mathrm{Cl}]$ \\
Experiment 1 & $2.66[2.51,2.82]$ & $0.09[-0.13,0.31]$ \\
Experiment 2 & $4.02[3.47,4.56]$ & $-0.82[-1.61,-0.03]$ \\
Experiment 3 & $3.49[2.87,4.11]$ & $-0.02[-0.98,0.94]$ \\
\hline
\end{tabular}

In this dataset, the linear regression and GP equations were both more accurate and less biased than the proportional model. The linear regression and GP models both performed similar to each other in an assessment of accuracy, though the linear regression model was slightly less biased. These models were then examined in a clinical sample to assess the accuracy of the equations in participants with elevated PTSD symptoms and the diagnostic sensitivity, specificity, and AUC of these scaled scores.

\section{Experiment 3}




\section{Methods}

\section{Participants}

Participants in this sample were 89 trauma exposed individuals who were either currently using or had a history of opioid use and endorsed a significant trauma history. Recruitment procedures for this study are described in [removed for blind review]. Participants' ages ranged from 20 to 72 years $(M=36.9, S D=10.19), 55.1 \%$ of the sample identified as male, $43.8 \%$ identified as female, and $1.1 \%$ identified as other. The majority of the sample (86.4\%) identified as White, 2.3\% identified as African American, 2.3\% identified as Asian American, 1.1\% identified as Pacific Islander/Native Alaskan, $4.5 \%$ as American Indian, and 3.4\% as Bi-Racial.

The majority of the sample identified as Not-Latino (96.4\%), and $88.8 \%$ reported an annual income of $\$ 30,000$ per year or less. About a quarter (29.2\%) of participants completed high school and $5.6 \%$ college or a higher level of education (Table 1). Participants in this study completed a battery of clinical interviews and self-report measures, including the LEC and PCL- 5 described previously in the original sample. Participants were eligible for this study if they met criteria for DSM-IV substance use criteria for opioids using the Addiction Severity Index lite (ASI-lite; McLellan, Cacciola, Zanis, 1997), identified opioids as their drug of choice, and were between 18-65 years of age. Participants were excluded if they endorsed active psychosis or did not speak English. One participant was excluded from these analyses as they did not have complete data on the PCL-8.

A score of 33 is the currently recommended cut-off indicating the likely presence of a PTSD (Bovin et al., 2016). Participants completed the Structured Clinical Interview for the DSM-5 (SCID-5; First, 2014) to assess current PTSD diagnosis. This SCID is a structured clinical interview that assess current symptom type and severity of diagnoses in the DSM-5. The sensitivity, specificity, and area under the curve (AUC) of each model was compared to that of the full PCL-5, in order to examine the accuracy in predicting PTSD diagnosis. Previous research (Price et al., 2016) found the full PCL5 to have an AUC of 0.72 and the PCL 8-item version to have an AUC of 0.71 . About two-thirds of the sample (66.3\%) met for a current diagnosis of PTSD based on the SCID-5.

\section{Results}

The performance of each model described previously was examined in this dataset for both absolute value, and bias (Table 3). The linear regression $\left(M_{\text {accuracy }}=3.42,95 \% \mathrm{Cl}[2.82,4.02]\right.$; $\left.M_{\text {bias }}=0.57,95 \% \mathrm{Cl}[-.37,1.50]\right)$ and GP $\left(M_{\text {accuracy }}=3.49,95 \% \mathrm{Cl}[2.87,4.11] ; M_{\text {bias }}=-0.02,95 \% \mathrm{Cl}\right.$ $[-0.98,0.94])$ models both performed more accurately than the proportional model ( $M_{\text {accuracy }}=4.49$, $\left.95 \% \mathrm{Cl}[3.75,5.23] ; M_{\text {bias }}=-3.22,95 \% \mathrm{Cl}[-4.22,-2.23]\right)$ in both accuracy and bias. However, the GP model demonstrated less bias than the linear regression and proportional models (Table 3 ).

Accuracy of a PTSD diagnosis based on a full PCL-5 score of 33 was examined by comparing the sensitivity, specificity, and AUC of the scaled score for each model to that of the full PCL-5 (Table 5). Each model was found to perform similarly to the full scale in terms of diagnostic utility.

\section{Discussion}

The present study examined three methods to convert scores from the abbreviated PCL-5 8 -item version (PCL-8) to the scale of the full PCL-5. The linear regression and GP models produced scores that were most similar to that of the full PCL- 5 in each sample. In the first dataset, these models produced scaled scores between 2-3 points from the original measure with the GP model being slightly superior to the linear regression model, in that the GP model demonstrated less 
Table 4. Multiple linear regression analysis of PCL-8 items

\begin{tabular}{lll}
\hline Variable & $\mathrm{B}$ & $\mathrm{SE}$ \\
Item 1 & $2.11 *$ & 0.18 \\
Item 2 & $1.82^{\star}$ & 0.14 \\
Item 3 & $1.54 *$ & 0.13 \\
Item 4 & $1.69 *$ & 0.13 \\
Item 5 & $2.92 *$ & 0.11 \\
Item 6 & $2.96 *$ & 0.13 \\
Item 7 & $2.59 *$ & 0.12 \\
Item 8 & $2.49 *$ & 0.13 \\
$R^{2}$ & 0.96 & \\
\hline
\end{tabular}

Table 5. Diagnostic accuracy of each model compared to the full PCL-5

\begin{tabular}{lccc}
\hline & Sensitivity at 33 & Specificity at 33 & AUC \\
Proportional Model & 0.88 & 0.70 & 0.80 \\
Linear Regression Model & 0.81 & 0.77 & 0.77 \\
GP Model & 0.85 & 0.73 & 0.79 \\
PCL-5 Total & 0.80 & 0.73 & 0.75 \\
\hline
\end{tabular}

bias than the linear regression model. In the two validation datasets (both clinical and community samples) these two models performed between 3-4.5 points from the original measure. The decline in performance is expected when moving to new data (Zou Hastie, 2005). These values were markedly improved from the scores obtained by scaling the 8 - item version by the number of items on the full PCL-5 (x 2.5) using a proportional model. These results suggest it is possible to use the abbreviated scale to obtain an accurate measure of symptoms on the same scale of a widely used measure of PTSD. Based on the performance across the three samples, it is recommended that the GP model is used.

These findings have important clinical and research implications. These scaling methods allow for accurate estimates of overall PTSD symptom severity to be obtained from repeated assessments at lower burden to participants. In EMA, the reduced length of the PTSD assessment allows for collection of other information, such as contextual variables including the presence of trauma or other relevant cues. The PCL-8 could be used as a daily assessment to measure type and severity of PTSD, while still utilizing the full PCL-5 score for assessing clinical severity. Indeed, in a recent qualitative study, providers reported a strong desire to check patient symptoms between sessions (Schueller, Washburn, Price, 2016). However, a concern with repeated assessment was the burden it would impose on patients. The use of a scaling method would allow for low-burden and interpretably assessments. Finally, all three models had comparable performance when used as a diagnostic screen.

The scaled score obtained by the GP model is best used as a method to approximate an individual's PTSD symptom severity within a narrow range. That is, one can expect the 'true' PCL-5 score to fall within +/-4.5 points of the scaled score. Care should be taken when making diagnostic or clinical decisions based on these scores as a result. Indeed, clinicians and researchers should consider the benefits afforded by the abbreviated scale against the psychometric costs. Specifically, does the reduction in administration time warrant the reduction in total score accuracy? Future studies should also examine the utility of these equations in an EMA collected sample, to determine if and how the bias and accuracy changes over time. 
This study had several limitations of note. These models were only assessed a community and substance using sample. It is unclear how these models would perform in other trauma exposed samples, such as military populations or victims of a specific type of trauma. Further, these models were assessed in a relatively small clinical sample. It is important to examine the accuracy and bias of these models in large clinical samples with a wide range of symptom severity and type of symptom.

Despite these limitations, the present study provides a method to convert an abbreviated PCL-5 to the scores of the full PCL-5. Such methods effectively use the PCL-8 with minimal loss of information from the full version. This approach opens the door to studies that would benefit from repeated assessment of PTSD symptoms.

\section{References}

Arroll, B., Goodyear-Smith, F., Crengle, S., Gunn, J., Kerse, N., Fishman, T., Falloon, K., and Hatcher, S. (2010). Validation of phq-2 and phq-9 to screen for major depression in the primary care population. The annals of family medicine, 8(4):348-353.

Blevins, C. A., Weathers, F. W., Davis, M. T., Witte, T. K., and Domino, J. L. (2015). The posttraumatic stress disorder checklist for dsm-5 (pcl-5): Development and initial psychometric evaluation. Journal of traumatic stress, 28(6):489-498.

Bryant, R. A., Creamer, M., O'Donnell, M., Forbes, D., McFarlane, A. C., Silove, D., and Hadzi-Pavlovic, D. (2017). Acute and chronic posttraumatic stress symptoms in the emergence of posttraumatic stress disorder: A network analysis. JAMA psychiatry, 74(2):135-142.

Dewey, D., McDonald, M. K., Brown, W. J., Boyd, S. J., Bunnell, B. E., and Schuldberg, D. (2015). The impact of ecological momentary assessment on posttraumatic stress symptom trajectory. Psychiatry Research, 230(2):300303.

Duhoux, A., Fournier, L., Gauvin, L., and Roberge, P. (2013). What is the association between quality of treatment for depression and patient outcomes? a cohort study of adults consulting in primary care. Journal of affective disorders, 151(1):265-274.

Edition, F. et al. (2013). Diagnostic and statistical manual of mental disorders. Am Psychiatric Assoc, 21.

First, M. B. (2014). Structured clinical interview for the dsm (scid). The encyclopedia of clinical psychology, pages $1-6$.

Gelkopf, M., Lapid Pickman, L., Carlson, E. B., and Greene, T. (2019). The dynamic relations among peritraumatic posttraumatic stress symptoms: An experience sampling study during wartime. Journal of traumatic stress, 32(1):119-129.

Kilpatrick, D. G., Resnick, H. S., Milanak, M. E., Miller, M. W., Keyes, K. M., and Friedman, M. J. (2013). National estimates of exposure to traumatic events and ptsd prevalence using dsm-iv and dsm-5 criteria. Journal of traumatic stress, 26(5):537-547.

Lang, A. J. and Stein, M. B. (2005). An abbreviated ptsd checklist for use as a screening instrument in primary care. Behaviour research and therapy, 43(5):585-594.

Löwe, B., Kroenke, K., and Gräfe, K. (2005). Detecting and monitoring depression with a two-item questionnaire (phq-2). Journal of psychosomatic research, 58(2):163-171.

Pereira-Lima, K., Loureiro, S. R., Bolsoni, L. M., Apolinario da Silva, T. D., and Osório, F. L. (2019). Psychometric properties and diagnostic utility of a brazilian version of the $\mathrm{pcl}-5$ (complete and abbreviated versions). European journal of psychotraumatology, 10(1):1581020.

Price, M., Brier, Z. M., Mirhashem, R., and Allen, H. C. (2018a). The impact of co-occurring opioid misuse and ptsd on response inhibition. Drug and alcohol dependence, 189:187-192.

Price, M., Legrand, A. C., Brier, Z. M., and Hébert-Dufresne, L. (2019). The symptoms at the center: examining the comorbidity of posttraumatic stress disorder, generalized anxiety disorder, and depression with network analysis. Journal of psychiatric research, 109:52-58. 
Price, M., Szafranski, D. D., van Stolk-Cooke, K., and Gros, D. F. (2016). Investigation of abbreviated 4 and 8 item versions of the ptsd checklist 5. Psychiatry research, 239:124-130.

Price, M., van Stolk-Cooke, K., Brier, Z. M., and Legrand, A. C. (2018b). mhealth solutions for early interventions after trauma: improvements and considerations for assessment and intervention throughout the acute posttrauma period. Mhealth, 4.

Price, M., Van Stolk-Cooke, K., Legrand, A. C., Brier, Z. M., Ward, H. L., Connor, J. P., Gratton, J., Freeman, K., and Skalka, C. (2018c). Implementing assessments via mobile during the acute posttrauma period: Feasibility, acceptability and strategies to improve response rates. European Journal of Psychotraumatology, 9(sup1):1500822.

Prins, A., Bovin, M. J., Smolenski, D. J., Marx, B. P., Kimerling, R., Jenkins-Guarnieri, M. A., Kaloupek, D. G., Schnurr, P. P., Kaiser, A. P., Leyva, Y. E., et al. (2016). The primary care ptsd screen for dsm-5 (pc-ptsd-5): development and evaluation within a veteran primary care sample. Journal of general internal medicine, 31(10):1206-1211.

Schmidt, M. and Lipson, H. (2009). Distilling free-form natural laws from experimental data. science, 324(5923):81-85.

Schueller, S. M., Washburn, J. J., and Price, M. (2016). Exploring mental health providers' interest in using web and mobile-based tools in their practices. Internet interventions, 4:145-151.

Schuler, K., Ruggero, C. J., Mahaffey, B., Gonzalez, A., L. Callahan, J., Boals, A., Waszczuk, M. A., Luft, B. J., and Kotov, R. (2021). When hindsight is not 20/20: ecological momentary assessment of ptsd symptoms versus retrospective report. Assessment, 28(1):238-247.

Shapiro, D., Jamner, L. D., Davydov, D. M., and James, P. (2002). Situations and moods associated with smoking in everyday life. Psychology of Addictive Behaviors, 16(4):342.

Shapiro, D. N., Chandler, J., and Mueller, P. A. (2013). Using mechanical turk to study clinical populations. Clinical psychological science, 1(2):213-220.

Shiffman, S., Stone, A., and Hufford, M. (2007). Ecological momentary assessment. annual review of clinical psychology.

van Stolk-Cooke, K., Brown, A., Maheux, A., Parent, J., Forehand, R., and Price, M. (2018). Crowdsourcing trauma: Psychopathology in a trauma-exposed sample recruited via mechanical turk. Journal of traumatic stress, 31(4):549-557.

Whelan, R. and Garavan, H. (2014). When optimism hurts: inflated predictions in psychiatric neuroimaging. Biological psychiatry, 75(9):746-748.

Wortmann, J. H., Jordan, A. H., Weathers, F. W., Resick, P. A., Dondanville, K. A., Hall-Clark, B., Foa, E. B., YoungMcCaughan, S., Yarvis, J. S., Hembree, E. A., et al. (2016). Psychometric analysis of the ptsd checklist-5 (pcl-5) among treatment-seeking military service members. Psychological assessment, 28(11):1392.

Zou, H. and Hastie, T. (2005). Regularization and variable selection via the elastic net. Journal of the royal statistical society: series $B$ (statistical methodology), 67(2):301-320. 